\title{
Study of the relaxation process during InGaAs/GaAs (001) growth from in situ real-time stress measurements
}

\author{
M. U. González, ${ }^{\text {a) }}$ Y. González, and L. González \\ Instituto de Microelectrónica de Madrid (CNM-CSIC), C/ Isaac Newton, 8 (PTM), 28760-Tres Cantos \\ (Madrid), Spain
}

(Received 19 February 2002; accepted 2 October 2002)

\begin{abstract}
Strain evolution during $\mathrm{In}_{0.2} \mathrm{Ga}_{0.8} \mathrm{As} / \mathrm{GaAs}$ (001) growth by molecular beam epitaxy has been monitored in real time. We have detected that three main relaxation stages, related to different mechanisms, take place during growth, and we have obtained the thickness range where those mechanisms are active. The in situ measured relaxation behavior in the plastic stages has been described by means of a simple equilibrium model that takes into account dislocations generation and interaction between them. The excellent agreement between the experimental data and the model allows us to determine the value of the formation energy per unit length of a misfit dislocation and the extent of the interaction between dislocations in this material system. (C) 2002 American Institute of Physics. [DOI: 10.1063/1.1524303]
\end{abstract}

Lattice mismatched heteroepitaxy constitutes a current approach in semiconductor technology. On one hand, fully strained layers are incorporated in optoelectronic devices taking advantage of the modification of the band structure by strain. On the other hand, relaxed buffer layers can be introduced to change substrate lattice parameter in order to achieve actual band-gap engineering without lattice parameter restrictions. Any of these applications requires the knowledge of strain evolution in the layers and the relaxation mechanisms involved.

Up until now, it has been clearly established for mismatched heteroepitaxial growth that, initially, the layer grows pseudomorphically and thus strained, accumulating elastic strain energy. With increasing accumulated elastic energy the layer becomes metastable, and it begins to relax at a critical thickness, $h_{c} .{ }^{1}$ Relaxation in strained layers takes place mainly through the formation of misfit dislocations, although elastic relaxation phenomena might be important either for thin enough layers or for growth conditions where dislocation formation is inhibited. Several mechanisms have been identified for the generation of misfit dislocations, such as bending of threading dislocations coming from the substrate, ${ }^{1}$ nucleation of new dislocations, ${ }^{2}$ and multiplication phenomena. ${ }^{3}$ Other studied processes appearing during relaxation are work hardening effects due to dislocations interaction. ${ }^{4-6}$ Different theoretical and empirical models ${ }^{1-7}$ have dealt with strain evolution, based on some of the aforementioned mechanisms and even incorporating kinetic effects. ${ }^{8}$ However, and despite the huge effort dedicated, the mechanisms involved during the relaxation process of III-V mismatched layers have not been fully determined yet and, consequently, there is no model able to predict the whole strain evolution. This could be partly due to experimental data scattering, since most of the experimental work has been done by measuring with different techniques the final strain state in many samples, for which differences (although small) in composition and growth conditions are unavoidable.

${ }^{a)}$ Electronic mail: ujue@imm.cnm.csic.es
In this letter, we present the strain evolution during the growth of $\mathrm{In}_{0.2} \mathrm{Ga}_{0.8} \mathrm{As} / \mathrm{GaAs}$ by molecular beam epitaxy (MBE), obtained in situ with a real-time stress measurement technique. ${ }^{9-11}$ This provides accurate experimental data for the whole relaxation process during the growth of a single sample. From these data, we have observed that three main stages related to different relaxation mechanisms take place during growth, and we have established the thickness range for those stages. First, an initial elastic relaxation regime, growth rate dependent, takes place. ${ }^{12}$ With increasing layer thickness, two new relaxation stages appear showing no kinetic effects associated with the growth rate. By means of a simple equilibrium model that involves dislocations generation and interaction between them, we have accurately described the relaxation evolution measured in situ for those two last stages and, from the fitting of our experimental data, we have determined the values of the formation energy of misfit dislocations and the dislocations interaction extent in this material system.

Samples were grown by MBE on on-axis Si-doped GaAs (001) epiready substrates with a nominal threading dislocation density of $10^{4} \mathrm{~cm}^{-2}$. After oxide thermal desorption, a $100 \mathrm{~nm}$ thick GaAs buffer layer was grown at substrate temperature $T_{s}=580^{\circ} \mathrm{C}$. The $\operatorname{In}_{x} \mathrm{Ga}_{1-x}$ As layers, with a nominal In content of $x=0.2$, were grown at $T_{s}=500^{\circ} \mathrm{C}$ using two different growth rates, 0.2 and 0.5 monolayers per second (ML/s). The in situ and real-time strain measurements were performed by following during growth the stressinduced substrate curvature through the deflection of a laser beam. ${ }^{9-11}$ For this purpose, the GaAs substrates were shaped as cantilevers and mounted on a special holder that allows them bending freely (see inset in Fig. 1 for measurement geometry). The substrate curvature, $\kappa$, is related to layer stress by the Stoney's equation: ${ }^{9} \Sigma \sigma=\left(\mathrm{M}_{\mathrm{s}} h_{s}^{2} \kappa\right) / 6$, where $h_{s}$ is the substrate thickness, $\mathrm{M}_{\mathrm{s}}$ is the biaxial modulus of the substrate and $\Sigma \sigma$, which we call layer accumulated stress, is the stress incorporated by the deposited material integrated over the layer thickness. From the accumulated stress, the average layer strain can be calculated $(\Sigma \sigma=\mathrm{M} \varepsilon h$, with $\varepsilon$ as the layer strain, $\mathrm{M}$ as the layer biaxial modulus, and $h$ as the 


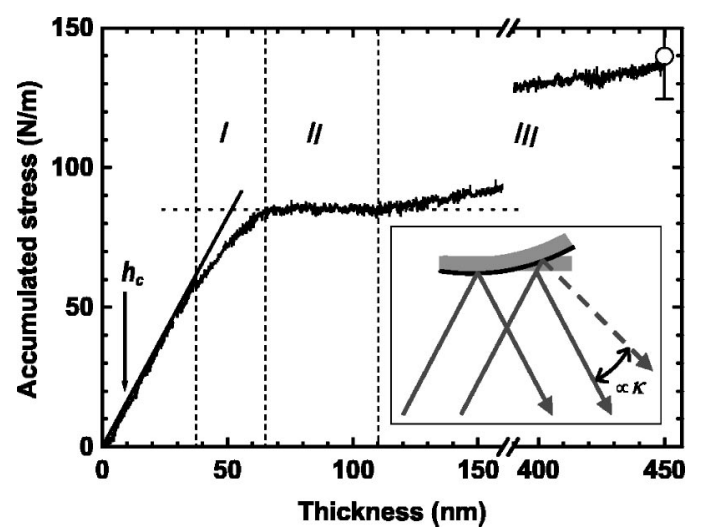

FIG. 1. In situ measured accumulated stress, $\Sigma \sigma$, during growth of $450 \mathrm{~nm}$ $\mathrm{In}_{0.2} \mathrm{Ga}_{0.8} \mathrm{As} / \mathrm{GaAs}$ by molecular beam epitaxy at $0.5 \mathrm{ML} / \mathrm{s}$. Dotted lines delimit the different relaxation stages. The continuous straight line represents pseudomorphic growth. The open circle is the final accumulated stress calculated from XRD measurements. The inset shows the measurement geometry.

layer thickness). ${ }^{9,11}$ In order to check the in situ obtained values, composition and final strain of the $\operatorname{In}_{0.2} \mathrm{Ga}_{0.8}$ As layers were also measured after growth with high-resolution $\mathrm{x}$-ray diffraction (XRD) technique.

Figure 1 shows the in situ measured accumulated stress, $\Sigma \sigma$, during growth of a $450 \mathrm{~nm}$ thick $\mathrm{In}_{0.2} \mathrm{Ga}_{0.8}$ As sample at $r_{g}=0.5 \mathrm{ML} / \mathrm{s}$. The data shown in Fig. 1 are, without any treatment, proportional to the in situ measured quantity. As we can see in Fig. 1, the accumulated stress increases linearly during the first $40 \mathrm{~nm}$. This corresponds to the initial pseudomorphic growth, where each deposited monolayer incorporates the amount of stress corresponding to the misfit strain, $\varepsilon_{0}$. We want to note that a thickness of $40 \mathrm{~nm}$ is well above Matthews critical thickness. ${ }^{1}$ However, the mechanism proposed by Matthews causes a very small relaxation (not detectable with the presently used measurement configuration) due to the limited number of sources, ${ }^{7,12}$ and then in this range pseudomorphic growth is still considered. From the initial accumulated stress slope, the composition of the InGaAs layer can be determined, being $x=0.2$ for the sample shown in Fig. 1. The values determined by postgrowth XRD measurements of In composition $(x=0.2)$ and final accumulated stress (open circle in Fig. 1) are in total agreement with the in situ obtained ones, ensuring the quantitative validity of the in situ measurements during the whole growth process.

As growth proceeds past $40 \mathrm{~nm}$, we observe a slope change in the accumulated stress, indicating the onset of relaxation. The evolution of $\Sigma \sigma$ curve from this point clearly shows three different behaviors, delimited by dashed lines in Fig. 1. From 40 to $65 \mathrm{~nm}, \Sigma \sigma$ increases in a nonlinear way; between 65 and $110 \mathrm{~nm}, \Sigma \sigma$ remains constant and from 110 $\mathrm{nm}$ on, it increases again in a quasilinear way. This indicates the existence of three stages in the relaxation process, with different mechanisms involved. Since both kinetic and equilibrium mechanisms have been considered during relaxation, ${ }^{1-8}$ we have performed stress measurements at different growth rates to discriminate between the two situations.

Figure 2 shows the strain evolution obtained for two InGaAs layers grown at 0.2 and $0.5 \mathrm{ML} / \mathrm{s}$. We have normalized the strain to the misfit value and the thickness to the Downloaded 14 Jun 2010 to 161.111.235.252. Redistribution subje

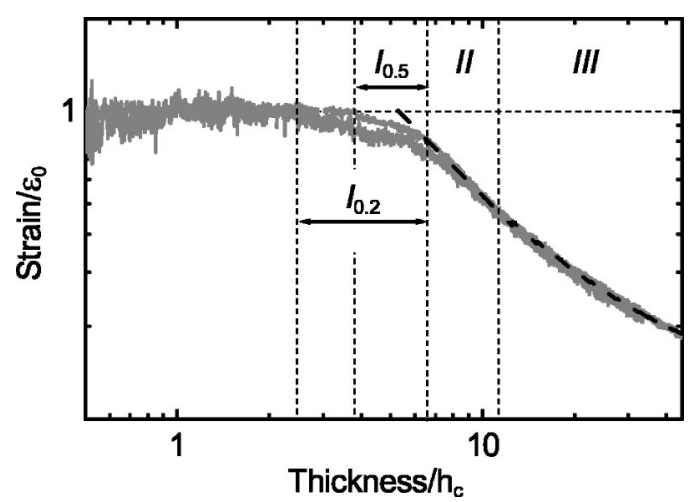

FIG. 2. Normalized strain evolution versus normalized thickness during growth of two $\mathrm{In}_{0.2} \mathrm{Ga}_{0.8} \mathrm{As} / \mathrm{GaAs}$ layers at two different growth rates, 0.2 and $0.5 \mathrm{ML} / \mathrm{s}$, obtained from in situ accumulated stress measurements. The dotted lines delimit the different relaxation stages (stage I is different for the two growth rates, $\mathrm{I}_{0.2} \neq \mathrm{I}_{0.5}$ ). The dashed line in stages II and III corresponds to the strain behavior obtained from Eq. (2).

Matthews critical thickness value for each layer in order to discard differences due to small changes in sample In composition. The dashed lines delimit the observed relaxation stages. The first stage of relaxation depends on the growth rate, as can be seen in Fig. 2. The onset of this stage changes with the particular growth conditions, but it is reproducibly around $40 \mathrm{~nm}$ for $0.5 \mathrm{ML} / \mathrm{s}$ and $30 \mathrm{~nm}$ for $0.2 \mathrm{ML} / \mathrm{s}$. Correlation of the stress measurements with also in situ morphology evolution data have allowed us to identify this as an elastic relaxation stage, as has been published elsewhere. ${ }^{12}$ In the other two stages, the strain behavior coincides for both growth rates even though the growth time is 2.5 times larger for the sample grown at $0.2 \mathrm{ML} / \mathrm{s}$ than for the other one. This means that the relaxation is not limited by kinetic constraints in those stages for the growth rates and substrate temperatures employed in this work, thus allowing us to consider the relaxation behavior there as an equilibrium process. This is to be expected for InGaAs/GaAs, since growth takes place at high temperatures relative to the brittle/ductile behavior transition, and then no kinetic constrains for dislocation velocities are expected ${ }^{3}$ it constitutes a main difference with the $\mathrm{SiGe} / \mathrm{Si}$ system, for which kinetic limitations are frequently found.

From now on, we will discuss the main mechanisms involved in the two last relaxation stages by using an equilibrium model. Fontaine et al. ${ }^{13}$ proposed a simple model that predicts $\Sigma \sigma$ behavior associated with dislocations formation. This model assumes that the total energy per unit area of the layer is $E=E_{\mathrm{el}}+E_{\mathrm{disl}}$, where $E_{\mathrm{el}}=\mathrm{M} h \varepsilon^{2}$ is the stored elastic energy and $E_{\text {disl }}=(2 / b) \xi_{0}\left(\varepsilon_{0}-\varepsilon\right)$ is the energy consumed by the dislocations needed to relax the layer from the misfit strain $\varepsilon_{0}$ to $\varepsilon$, assuming that all dislocations are of the $60^{\circ}$ type. $\xi_{0}$ is the formation energy of a new dislocation per unit length and $b$ is the Burgers vector of the dislocations. Then, minimizing the total energy at a given thickness, we obtain:

$$
\Sigma \sigma=\mathrm{M} \varepsilon h=\xi_{0} b=\text { constant. }
$$

This is the behavior we have found in our layers between 65 and $110 \mathrm{~nm}$ (see Fig. 1). So, we can associate this stage (stage II) in relaxation with the formation of new dislocations, and strain evolution here can be described under the to AIP license or copyright; see http://apl.aip.org/apl/copyright.jsp 
simple law $\varepsilon h=$ constant. This result was also obtained by Dunstan et al. ${ }^{3,14}$ based on geometrical considerations instead of energetic arguments. They found, after postgrowth characterization of a large number of samples, the empirical law $\varepsilon h=0.8 \pm 0.1 \mathrm{~nm}$ for the InGaAs case. ${ }^{3}$ In our case, the constant value, more accurately determined, is $0.75 \mathrm{~nm}$ for $\mathrm{In}_{0.2} \mathrm{Ga}_{0.8} \mathrm{As} / \mathrm{GaAs}$ (001). The value we obtain for the formation energy of a dislocation is $\xi_{0}=3.4 \times 10^{-8} \mathrm{~J} / \mathrm{m}$, in good agreement with theoretically calculated values. ${ }^{13}$ Misfit dislocations can be generated either by nucleation of half loops or by multiplication of previously existing dislocations, but our present measurements cannot provide any information on the predominant mechanism.

As growth proceeds, we can see in Figs. 1 and 2 that the $\Sigma \sigma$ and $\varepsilon$ behaviors diverge from the previous law, showing a reduction in the relaxation rate. Several models have considered interaction between dislocations in layers with high dislocation density as responsible for these kinds of effects, related to work hardening phenomena. ${ }^{4-6}$ To interpret our experimental results, we have extended the above described model by introducing a new term in the formation energy per unit length of a dislocation, which accounts for the interaction of the dislocation with the dislocation array already present in the layer: $\xi=\xi_{0}+\xi_{\text {int }}$. $\xi_{\text {int }}$ depends on the distance between dislocations and on the layer thickness, ${ }^{4-6}$ and for large thickness and high dislocation density it can be expressed as ${ }^{5,6} \xi_{\text {int }}=A\left(\varepsilon_{0}-\varepsilon\right) h$, where $A$ is a constant. In order to use this expression for lower density and smaller thickness, we have combined it with Dodson's approach ${ }^{4}$ that considers the dislocation interaction extent. Within this approach, $A$ is not a constant but $A=A^{\prime} S_{n}$, with $S_{n}$ $=\sum_{i=1}^{n} 1 / i, n$ being the number of dislocations interacting, which depends on the interaction extent. Although Dodson took the interaction extent to be the layer thickness, we have left it as a fitting parameter. In fact, we do not need to assume any value for this parameter since we have continuous experimental data of $\varepsilon$ evolution during the whole relaxation process, and the value can thus be determined from the transition point between stages II and III. Then, the total energy of the layer is: $E=\mathrm{M} \varepsilon^{2} h+\left(\xi_{0}+\xi_{\text {int }}\right)(2 / b)\left(\varepsilon_{0}-\varepsilon\right)$, and after minimization, we obtain:

$$
\varepsilon h=\left(1+\frac{2 A^{\prime} S_{n}}{\mathrm{Mb}}\right)^{-1}\left[\frac{\xi_{0}}{\mathrm{Mb}}+\frac{2 A^{\prime} S_{n}}{\mathrm{Mb}} \varepsilon_{0} h\right]
$$

By fitting $A^{\prime}$ and $n$ (using our obtained value $\xi_{0}=3.4$ $\times 10^{-8} \mathrm{~J} / \mathrm{m}$ ), the strain obtained from the in situ measurements can be described with this equation for both stages II and III. The dashed line plotted in Fig. 2 shows the good agreement between the measured strain values and Eq. (2). From the $n$ fitting value, we have obtained the dislocation interaction extent to be $0.6 \mathrm{~h}$.

Summarizing, we have performed in situ stress measurements during the growth of InGaAs/GaAs (001) layers. From the accumulated stress behavior measured, we can distinguish different stages in the relaxation process and determine their thickness range. We have developed a simple equilibrium model that describes the experimental data obtained in the plastic relaxation regimes. This model allows us to obtain the value of the formation energy of a misfit dislocation and the dislocations interaction extent in this material system.

The authors would like to acknowledge F. Briones, J. P. Silveira, and J. M. García for their help with the experimental setup for the in situ measurements and valuable discussions. This work was supported by Spanish "CICYT" under Project No. MAT2000-1625. One of the authors (M.U.G.) thanks the Consejería de Educación y Cultura de la Comunidad de Madrid for funding.

${ }^{1}$ J. W. Matthews and A. E. Blakeslee, J. Cryst. Growth 27, 118 (1974).

${ }^{2}$ P. M. J. Marée, J. C. Barbour, J. F. van der Veen, K. L. Kavanagh, C. W. T. Bulle-Lieuwma, and M. P. A. Viegers, J. Appl. Phys. 62, 4413 (1987).

${ }^{3}$ D. J. Dunstan, P. Kidd, R. Beanland, A. Sacedón, E. Calleja, L. González, Y. González, and F. J. Pacheco, Mater. Sci. Technol. 12, 181 (1996).

${ }^{4}$ B. W. Dodson, Appl. Phys. Lett. 53, 37 (1988).

${ }^{5}$ T.-Y. Zhang, J. E. Hack, and L. J. Guido, J. Appl. Phys. 75, 2363 (1994).

${ }^{6}$ D. González, D. Araújo, G. Aragón, and R. García, Appl. Phys. Lett. 72, 1875 (1998)

${ }^{7}$ V. Drigo, A. Aydinli, A. Carnera, F. Genova, C. Rigo, C. Ferrari, P. Franzosi, and G. Salviati, J. Appl. Phys. 66, 1975 (1989).

${ }^{8}$ B. W. Dodson and J. Y. Tsao, Appl. Phys. Lett. 51, 1325 (1987).

${ }^{9}$ J. A. Floro, E. Chason, S. R. Lee, R. D. Twesten, R. Q. Hwang, and L. B. Freund, J. Electron. Mater. 26, 969 (1997).

${ }^{10}$ J. M. García, J. P. Silveira, and F. Briones, Appl. Phys. Lett. 77, 409 (2000).

${ }^{11}$ R. Beresford, K. Tetz, J. Yin, E. Chason, and M. U. González, J. Vac. Sci. Technol. B 19, 1572 (2001).

12 M. U. González, Y. González, and L. González, Appl. Surf. Sci. 188, 128 (2002).

${ }^{13}$ C. Fontaine, J. P. Gailliard, S. Magli, A. Million, and J. Piaguet, Appl. Phys. Lett. 50, 903 (1987).

${ }^{14}$ D. J. Dunstan, S. Young, and R. H. Dixon, J. Appl. Phys. 70, 3038 (1991). 